\title{
A NICER SPECTRUM OF MAXI J1535-571: NEAR-MAXIMAL BLACK HOLE SPIN AND POTENTIAL DISK WARPING
}

\author{
J. M. Miller ${ }^{1}$, K. Gendreau ${ }^{2}$, R. M. Ludlam ${ }^{1}$, A. C. Fabian ${ }^{3}$, D. Altamirano ${ }^{4}$ Z. Arzoumanian ${ }^{2}$, P. M. Bult ${ }^{2}$, \\ E. M. CACKETT ${ }^{5}$, J. HOMAN ${ }^{6,7}$, E. KARA ${ }^{8}$, J. NEILSEN ${ }^{9}$, R. A. REMILLARD ${ }^{10}$, F. TOMBESI ${ }^{8,2,11,12}$
}

\begin{abstract}
We report on a NICER observation of the Galactic X-ray binary and stellar-mass black hole candidate, MAXI J1535-571. The source was likely observed in an "intermediate" or "very high" state, with important contributions from both an accretion disk and hard X-ray corona. The 2.3-10 keV spectrum shows clear hallmarks of relativistic disk reflection. Fits with a suitable model strongly indicate a near-maximal spin parameter of $a=c J / G M^{2}=0.994(2)$ and a disk that extends close to the innermost stable circular orbit, $r / r_{I S C O}=1.08(8)$ ( $\sigma$ statistical errors). In addition to the relativistic spectrum from the innermost disk, a relatively narrow Fe $\mathrm{K}$ emission line is also required. The resolution of NICER reveals that the narrow line may be asymmetric, indicating a specific range of emission radii. Fits with a relativistic line model suggest an inner radius of $r=144_{-60}^{+140} G M / c^{2}$ for the putative second reflection geometry; full reflection models suggest that radii a few times larger are possible. The origin of the narrow line is uncertain but a warp likely provides the most physically plausible explanation. We discuss our results in terms of the potential for NICER to reveal new features of the inner and intermediate accretion disk around black holes.
\end{abstract}

\section{INTRODUCTION}

MAXI J1535-571 was discovered as a new X-ray source by MAXI on 2017 September 02 (Negoro et al. 2017a; also see Nakahira et al. 2018). The source displays many X-ray spectral and timing features consistent with $\mathrm{X}$-ray binaries that harbor a stellar-mass black hole, including quasi-periodic oscillations (QPOs) and a relativistic $\mathrm{Fe} \mathrm{K}$ line suggestive of a high black hole spin parameter $\left(a=0.88_{-0.20}^{+0.10}\right.$, where $a=c J / G M^{2}$; Gendreau et al. 2017). No Type-I X-ray bursts have been observed from MAXI J1535-571, nor have any coherent pulsations or $\mathrm{kHz}$ quasi-periodic oscillations (Negoro et al. 2017b, Gendreau et al. 2017); positive detections of pulsations or $\mathrm{kHz}$ QPOs would signal a neutron star primary. The radio flux of the source also signals that it is likely a black hole (Russell et al. 2017); neutron stars tend to have a lower radio/X-ray ratio than stellar-mass black holes (e.g., Migliari \& Fender 2006).

The interstellar absorption along the line of sight to MAXI J1535-571 appears to be relatively high. Values of $N_{H}=3.6 \pm$ $0.2 \times 10^{22} \mathrm{~cm}^{-2}$ have been reported (Kennea et al. 2017). Guver \& Ozel (2009) find that $N_{H}\left(\mathrm{~cm}^{-2}\right)=2.21 \pm 0.09 \times 10^{21} A_{V}$

\footnotetext{
${ }^{1}$ Department of Astronomy, University of Michigan, 1085 South University Avenue, Ann Arbor, MI 48109-1107, USA, jonmm@umich.edu

${ }^{2}$ NASA Goddard Space Flight Center, Code 662, Greenbelt, MD, 20771, USA

${ }^{3}$ Institute of Astronomy, University of Cambridge, CB3 OHA, UK

${ }^{4}$ Department of Physics \& Astronomy, University of Southampton, Southampton, Hampshire S017 1BJ, UK

${ }^{5}$ Department of Physics \& Astronomy, Wayne State University, $666 \mathrm{~W}$. Hancock Street, Detroit, MI, 48201, USA

${ }^{6}$ Eureka Scientific, Inc., 2452 Delmer Street, Oakland CA 94602, USA

${ }^{7}$ SRON, Netherlands Institute for Space Research, Sorbonnelaan 2, 3584 CA Utrecht, The Netherlands

8 Department of Astronomy, University of Maryland, College Park, MD 20742, USA

${ }^{9}$ Department of Physics, Villanova University, Villanova, PA, 19085, USA

${ }^{10}$ MIT Kavli Institute for Astrophysics and Space Research, 70 Vassar Street, Cambridge, MA 02139, USA

${ }^{11}$ Department of Physics, University of Rome "Tor Vergata", Via della Ricerca Scientifica 1, I-00133 Rome, Italy

${ }^{12}$ INAF Astronomical Observatory of Rome, Via Frascati 33, 00078 Monteporzio Catone, Italy
}

(mag), this implies $A_{V} \simeq 16$. Standard optical methods of measuring the mass function may be very difficult for MAXI J1535-571.

The most complete prior X-ray analysis of MAXI J1535-571 was made by Xu et al. (2018), based on a NuSTAR exposure. The spectrum obtained by NuSTAR shows strong signatures of $\mathrm{X}$-ray reflection from the inner disk, and constrain the black hole spin parameter to be $a \geq 0.84$. This spin is broadly consistent with an initial report from Gendreau et al. (2017), who made preliminary fits to a NICER observation of MAXI J1535-571 immediately preceding the case analyzed in this work. The spin of the black hole in this X-ray binary may be the parameter of greatest interest and importance, but the presence of an additional narrow Fe K emission line is also worthy of note.

The broad passband, spectral resolution, sensitivity, and throughput of NuSTAR have made it an excellent tool for measuring black hole spin (see, e.g., Miller et al. 2013, Tomsick et al. 2014, El Batal et al. 2016). NICER (Gendreau et al. 2012) has a narrower passband, but its spectral resolution is roughly two times sharper ( $\Delta E \simeq 137 \mathrm{eV}$ at $6 \mathrm{keV})$, and it is suited to monitoring observations in a manner that is reminiscent of $R X T E$. In the near future, NICER may contribute significantly to efforts aimed at measuring black hole spin and revealing the geometry of the innermost accretion flow through its monitoring capability, not merely through single observations.

\section{OBSERVATIONS AND DATA REDUCTION}

We consider NICER observation 1050360106, which started on 2017 September 13 at 23:58:25 (UT). The data were processed using NICER software version 2018-0222 V002d. The data were screened to omit times of passage through the South Atlantic Anomaly (SAA) and pointing offsets greater than $54^{\prime \prime}$, and to only accept data obtained with an Earth angle $\geq 30^{\circ}$ above dark limb and $\geq 45^{\circ}$ above bright limb. After creating GTIs based on these criteria, NIEXTRACT-EVENTS was used to select events with a PI value between 20 and $1200(0.20-12.0 \mathrm{keV}$, the nominal NICER passband) and using "EVENT_FLAGS=bx 1x000".

This procedure resulted in a net exposure of 5112 seconds, and a nominal average count rate of 4203 counts $s^{-1}$. In the 
6.0-7.0 keV band, the average count rate is 187 counts $\mathrm{s}^{-1}$. NICER is not an imaging instrument, so backgrounds must be constructed from fields observed for that purpose. Bult et al. (2018) estimated the NICER background at 1.5 counts s${ }^{-1}$ in the $0.4-10.0 \mathrm{keV}$ band. Different background regions, or combinations of regions, might result in a rate that is a few times higher, but this is still a small fraction of the count rate observed from MAXI J1535-571. Background subtraction can be important at much lower count rates, and especially at low energy. Owing to the very high count rate observed from MAXI J1535-571 and its high interstellar column, we have not subtracted a background.

\section{ANALYSIS \& RESULTS}

The spectrum was analyzed using XSPEC version 12.10.0 (Arnaud 1996), using NICER instrument response files (version 1.02) and standard data weighting. The extremely bright nature of MAXI J1535-571 obviated any need for rebinning prior to analysis within XSPEC. The fitting procedure minimized the $\chi^{2}$ goodness-of-fit statistic. Unless otherwise noted, the errors quoted in this work are based on the values of a given parameter on the boundary of its $1 \sigma$ confidence interval. More conservative errors are sometimes quoted for continuum spectral fits, but $1 \sigma$ errors are standard for line spectroscopy since this permits direct estimates of line significance; our analysis is a combination of continuum and line spectroscopy.

Calibration residuals remain in the Si band (conservatively, 1.7-2.1 keV) owing to sharp changes in the instrumental sensitivity curve. Additional strong residuals remain that are likely tied to Au edges from the mirror coatings at and around 2.2-âĂŞ2.3 keV. These issues are typical for X-ray missions and Si-based detectors, especially in the early phase of any mission, but are particularly noticeable here due to NICERâÁŹs high soft X-ray throughput. We set a lower energy bound of $2.3 \mathrm{keV}$ for our spectral analysis. Though the spectrum might nominally be extended up to $12 \mathrm{keV}$, the upper range of any detector band is particularly difficult to calibrate, and we set an upper threshold of $10.0 \mathrm{keV}$. As the mission calibration is refined, it may be practical to model the data down to $0.5 \mathrm{keV}$ and up to $12 \mathrm{keV}$.

We initially explored fits with absorbed single-component models, including a multi-color disk (di skbb, Mitsuda et al. 1984) and power-law functions. The line-of-sight absorption was characterized in terms of an equivalent neutral hydrogen column density via the tbabs model (Wilms, Allen, \& McCray 2000). These models are rejected by the data (powerlaw: $\chi^{2} / \nu=17.0$, disk blackbody: $\chi^{2} / \nu=10.8$, where $\nu$ is the number of degrees of freedom). A significantly improved fit is obtained when these components are combined $\left(\chi^{2} / \nu=3.1\right)$; the residuals are consistent with calibration uncertainties below $E \leq 3 \mathrm{keV}$ and broad Fe K emission, signaling reflection from the inner disk (see Figure 1). In these fits, there is evidence of an edge feature at $E \simeq 9 \mathrm{keV}$. It is not fully consistent with the K-shell edges of Fe XXV or Fe XXVI $(8.83 \mathrm{keV}$ and $9.28 \mathrm{keV}$, respectively), nor with $\mathrm{Au}$ L-shell edges (9.63 keV and $9.71 \mathrm{keV}$ ) that could be instrumental. The feature is weak and does not affect the measurements of black hole spin made below.

Strong disk reflection is expected based on the prior reports from Gendreau et al. (2017) and Xu et al. (2018). We therefore proceeded to make fits with a model consisting of lineof-sight absorption in the ISM, a disk blackbody component, a relativistically blurred reflection model (relxill, which includes a cut-off power-law; Garcia et al. 2014, Dauser et al. 2014), and a photoelectric absorption edge. The relxill model assumes a broken power-law emissivity function (for $r \leq r_{b r}, j \propto r^{-q_{\text {in }}}$; for $r \geq r_{b r}, j \propto r^{-q_{\text {out }}}$, where $j$ is the emissivity); following recent work motivated by ray-tracing studies, we bounded the inner emissivity in the range $3 \leq q_{\text {in }} \leq 10$, the outer emissivity in the range $0 \leq q_{\text {out }} \leq 3$, and the break radius in the range $2 G M / c^{2} \leq r_{b r} \leq 6 G M / c^{2}$ (see Wilkins \& Fabian 2012; also see Miller et al. 2013, Tomsick et al. 2014). Owing to the narrow fitting range, we arbitarily fixed the power-law cut-off energy at its default value of $E_{\text {cut }}=300 \mathrm{keV}$.

This model yielded a vastly improved description of the data $\left(\chi^{2} / \nu=989.6 / 752=1.32\right)$. This fit is not formally acceptable, largely owing to the small calibration uncertainties that remain in the $2.3-3 \mathrm{keV}$ range; even $1-2 \%$ residuals become significant at the count rates observed from MAXI J1535-571. However, there is also evidence of a (comparatively) narrow Fe $\mathrm{K}$ emission line in the 6.6-6.7 keV range that is not modeled by reflection from close to the ISCO (see Figures 2 and 3). After rebinning heavily, the line appears to be asymmetric (if it is a single line), as per a (less) relativistic line emerging from a range of radii outside of the ISCO. We therefore made fits including the stand-alone relline model (the basis of the blurring function within relxill). We also explored fits with a simple Gaussian function, and combinations of three Gaussians with linked velocity widths, and centroids corresponding to the He-like Fe XXV triplet. This might correspond to lines produced in a disk wind viewed at an intermediate inclination (see, e.g., Miller et al. 2015, 2016).

The best model is the one that fits the (comparatively) narrow $\mathrm{Fe} \mathrm{K}$ emission line with a relline function $\left(\chi^{2} / \nu=\right.$ $944.3 / 747=1.264)$; the parameters of this model are listed in Table 1 (also see Figure 2, and Figure 3). Fits with a single Gaussian are significantly worse $\left(\chi^{2} / \nu=969.0 / 749=1.322\right.$, excluded at the $4.0 \sigma$ level via an F-test). The triplet of Gaussians with linked widths and line centroids corresponding to He-like Fe XXV is not rigidly excluded $\left(\chi^{2} / \nu=951.2 / 748=\right.$ 1.326 , worse than the best-fit model at the $2.3 \sigma$ level of confidence).

In our best-fit model with relxill, the spin of the black hole is tightly constrained: $a=0.994(2)$. This is broadly consistent with the results reported by Gendreau et al. (2017), and results from an independent spectrum obtained with $\mathrm{NuS}$ $T A R$ (Xu et al. 2018). The inner edge of the accretion disk is measured to sit at a radius consistent with the ISCO: $r_{i n}=$ 1.08(8) $r_{I S C O}$. Other parameters critical to the reflection spectrum are also well determined: the inner emissivity index is measured to be $q_{\text {in }}=7.8(4)$, and the outer emissivity index is measured to be $q_{\text {out }}=1.40_{-0.19}^{+0.08}$. This profile is broadly consistent with theoretical predictions of a very compact corona at a low scale height above the black hole (e.g., Wilkins \& Fabian 2012; also see Miller et al. 2013). The measured reflection fraction of $f_{\text {refl }}=2.65_{-0.18}^{+0.05}$ indicates that the observed spectrum is reflection-dominated; this is also consistent with a compact corona close to the black hole. Were a coronal source more distant from the black hole, light bending would be less severe, and more direct emission would be observed.

Although the results of fits made using the most basic relxill model strongly suggest a compact corona, the inferred corona may differ from a true "lamp-post" geometry ("lamp-post" versions of relxill produce significantly 
worse fits: $\chi^{2} / \nu=1062.0 / 749$, excluded at more than $8 \sigma$ via an F-test). This may be echoed by the "break" radius of the emissivity function, $r_{b r}=5.8(2) G M / c^{2}$; the radius is somewhat larger than the value measured in other sources, and may indicate a corona that is more radially extended. The best-fit model also indicates a fairly high disk temperature of $k T=2.16(4) \mathrm{keV}$, and a very small emitting area, though both are broadly consistent with values measured in "intermediate" and "very high" states in some sources (e.g., Sobczak et al. 1999). The power-law index, $\Gamma=2.303(5)$, also indicates that the source was likely observed in such a state. Of course, the measured values are the color temperature and area, and standard correction factors would imply a lower temperature and larger emitting area (e.g., Sobczak et al. 1999, Park et al. 2004).

The results of fits with relline are of particular interest. The inner radius of the line production region is measured to be $r_{i n}=144_{-60}^{+140} G M / c^{2}$. At such radii, the emissivity is expected to trend toward the Euclidian value of $q=3$ (Wilkins $\&$ Fabian 2012); this parameter is not tightly constrained, but is measured to be consistent with this: $q=2.65_{-0.65}^{1.35}$. The inclination of the narrow line is measured to be $\theta=37_{-13}^{+22}$ degrees; nominally, this differs from the inclination of the inner disk measured with relxill, $\theta=67.4(8)$ degrees. This is potentially interesting as differing inclinations may signal a warp, but the $90 \%$ confidence errors on the inclination include the value from the innermost disk.

The "narrow" Fe $\mathrm{K}$ emission line is weak, with a measured equivalent width of $W=10(1) \mathrm{eV}$. Yet, in this NICER spectrum, the line is required at the $7.5 \sigma$ level of confidence, as measured by dividing the line normalization by its $1 \sigma$ minus-side error. Replacement of the relline model with an unblurred reflection model, xillver (the core of the relxill), gave a significantly worse fit $\left(\chi^{2} / \nu=\right.$ $1116.6 / 747.0=1.495$, excluded at more than the $8 \sigma$ level of confidence). This establishes that the line broadening is dominated by dynamics, not scattering.

Modeling with two full relativistic reflection components can potentially account for scattering and give an improved estimate of the radius at which the secondary reflection occurs. We therefore explored fits with two full relativistic reflection models, including the basic relxill and versions of that model suited to a Comptonization continuum (relxillcp) and a high-density disk (relxilld). The electron temperature of the corona is not well constrained via the Comptonization model. The density-sensitive models prefer a number density of $n \simeq 10^{18} \mathrm{~cm}^{-3}$ in the inner disk, slightly lower than recently reported in the neutron star binary Serpens X-1 (Ludlam et al. 2018). In all cases, the inner disk radius remains consistent with the ISCO and the spin remains very high, $a \geq 0.96$. These models are generally worse by $\Delta \chi^{2} \simeq 20-50$ than the model in Table 1, but they all return inner radii for the second reflector in the range of $r=100-900 \mathrm{GM} / \mathrm{c}^{2}$ within $90 \%$ confidence.

This effort revealed that there are numerous ways by which two full relativistic reflection models might be linked or separated; the physical implications of these choices are complex and nuanced. A full treatment of these matters is beyond the scope of this paper, but the issues are important, especially at the sensitivity achieved with NICER. A detailed treatment is deferred to a later paper.

\section{DISCUSSION}

We have analyzed an early NICER observation of the bright black hole candidate, MAXI J1535-571. The source was likely observed in an "intermediate" or "very high" state, based on the observed parameters of the continuum spectrum. Very strong reflection from an accretion disk extending to the ISCO is observed, and the best-fit model measures a very high spin parameter: $a=0.994(2)$. This value is compatible with prior reports (Gendreau et al. 2017, Xu et al. 2018). Relativistically-shaped disk reflection spectra - including characteristic Fe K emission lines - are common in stellar-mass black holes; NICER has enabled the simultaneous detection and study of a narrower Fe $\mathrm{K}$ line. The shape of the line is likely dominated by dynamics, and simple fits suggest that it may originate as close as $r=144_{-60}^{+140} G M / c^{2}$; other fits suggest that larger radii remain possible. In this section, we discuss some of the strengths and weaknesses of our spectral modeling, implications for the innermost and intermediate accretion disk, and the potential of future NICER observations and studies.

The spin of MAXI J1535-571 was measured using the relxill model (Dauser et al. 2014, Garcia et al. 2014), which is now the standard for disk reflection modeling and spin constraints. The unusual precision of the spin measurement reflects the sensitivity that can be achieved using NICER. However, we caution that systematic uncertainties are likely to be much larger than the quoted statistical errors. Spin measurements using the accretion disk depend on the extent to which the optically-thick disk is truncated at the test-particle ISCO, making this the most important source of systematic errors. Currently, this question can only be explored using numerical simulations of accretion disks; for now, at least, these efforts suggest that fluid disks respect the test particle ISCO for $L \leq 0.3 L_{E d d}$. (e.g., Reynolds \& Fabian 2008; Shafee, Narayan, \& McClintock 2008). Remaining errors in the energy calibration of NICER are about $30 \mathrm{eV}$; since the spin is primarily derived from the shift of the red wing of the Fe $\mathrm{K}$ line, this roughly translates to a $0.4 \%$ systematic error on the spin parameter.

As the distance to MAXI J1535-571 and the mass of the black hole primary are unknown, we cannot estimate the Eddington fraction at which this NICER observation was made. However, the fact that a relativistic line characteristic of the innermost disk is clearly detected signals that matter has not been ejected into the line of sight, as per the case of the likely super-Eddington outburst of V404 Cyg (e.g., King et al. 2015). Moreover, clear QPOs are contemporaneously detected with coherence values of $Q=6$ (Gendreau et al. 2017); scattering in a dense outflow would lead to different light travel times and would de-cohere frequencies produced at smaller radii.

Although it is weak, the relatively narrow $\mathrm{Fe} \mathrm{K}$ line detected between $6.6-6.7 \mathrm{keV}$ may be particularly important. A similar feature was detected with NuSTAR (Xu et al. 2018), but it was not treated with dynamical broadening. Re-emission in a disk wind could potentially explain this emission. However, winds are typically detected in "high/soft" or "thermal-dominant" states (see, e.g., Ponti et al. 2012), rather than the "intermediate" or "very high" state sampled by this spectrum. Moreover, there is no evidence of wind absorption in this spectrum, though winds are likely equatorial and MAXI J1535-571 appears to be viewed at a high inclination.

Our analysis favors an emission line shaped primarily by Doppler shifts (and, weak gravitational redshifts), nominally 
at an intermediate radius of $r_{i n}=144_{-60}^{+140} G M / c^{2}$. Preliminary fits with full reflection models allow for radii that are larger by a factor of a few. The modeling results are likely best explained in terms of a warp that locally alters the profile of the accetion disk, causing more incident radiation to be reflected. It is not clear if simple radiation-driven warps can persist so close to a black hole (e.g., Pringle 1996, Maloney \& Begelman 1997). Other means of changing the disk profile exist; for instance, gravitomagnetic modes can give rise to a "corrugated" disk surface and potentially QPOs (e.g., Markovic \& Lamb 1998).

Low-frequency QPOs like those contemporaneously detected in MAXI J1535-571 (Gendreau et al. 2017) may not be Keplerian; at minimum, their energy dependence indicates that they are unlikely to be produced through local dissipation in such orbits. Reflection from Keplerian structures at intermediate disk radii - producing the narrow $\mathrm{Fe} \mathrm{K}$ line in MAXI J1535-571 - could connect the central engine to distant Keplerian time scales. Links between disk reflection spectra and QPOs have been reported previously, and can potentially be explained in terms of Lense-Thirring precession (see, e.g., Miller \& Homan 2005; Miller, Homan, \& Schnittman 2006; Ingram \& Done 2012; Ingram et al. 2016, 2017).

We are grateful to the anonymous referee for a helpful review of this paper. JMM acknowledges discussions with Phil Uttley.

\section{REFERENCES}

Arnaud, K., 1996, in ASP Conf. Ser. 101, Astronomical Data Analysis Software Systems V, ed. G. H. Jacoby \& J. Barnes (San Francisco, CA: ASP), 17

Bult, P., Arzoumanian, Z., Cackett, E., et al., 2018, ApJ, 859, L1

Dauser, T., Garcia, J., Parker, M., Fabian, A., Wilms, J., 2014, MNRAS, 444, L100

El Batal, A. M., Miller, J. M., Reynolds, M. T., et al., 2016, ApJ, 826, L12

Garcia, J., Dauser, T., Lohfink, A., Kallman, T., Steiner, J., McClintock, J., Brenneman, L., Wilms, J., Eikmann, W., Reynolds, C., Tombesi, F., 2014, ApJ, 782, 76

Gendreau, K., Arzoumanian, Z, \& Okajima, T., 2012, in Society of PhotoOptical Instrumentation Engineers (SPIE) Conference Series, Vol. 8443

Gendreau, K., Arzoumanian, Z., Markwardt, C., et al., 2017, ATEL 10768

Guver, T., \& Ozel, F., 2009, MNRAS, 400, 2050

Ingram, A., \& Done, C., 2012, MNRAS, 427, 934

Ingram, A., van der Klis, M., Middleton, M., Done, C., Altamirano, D., Heil, L., Uttley, P., Axelsson, M., 2016, MNRAS, 461, 1967

Ingram, A., van der Klis, M., Middleton, M., Altamirano, D., Uttley, P., 2017, MNRAS, 464, 2979

Kennea, J. A., Evans, P. A., Beardmore, A. P., Krimm, H. A., Romano, P., Yamaoka, K., Serino, M., Negoro, H., 2017, ATEL 10700

King, A. L., Miller, J. M., Raymond, J., Reynolds, M. T., Morningstar, W., 2015, ApJ, 813m, L37

Markovic, D., \& Lamb, F. K., 1998, ApJ, 507, 316

Migliari, S., \& Fender, R., 2006, MNRAS, 366, 79

Miller, J. M., \& Homan, J., 2005, ApJ, 618, L107
Miller, J. M., Parker, M. L., Fuerst, F., et al., 2013, ApJ, 775, L45

Miller, J. M., Fabian, A. C., Kaastra, J., Kallman, T., King, A. L., Proga, D., Raymond, J., Reynolds, C. S., 2015, ApJ, 814, 87

Miller, J. M., Raymond, J., Fabian, A. C., Gallo, E., Kaastra, J., Kallman, T., King, A. L., Proga, D., Reynolds, C., Zoghbi, A., 2016, ApJ, 821, L9

Mitsuda, K., Inoue, H., Koyama, K., Makishima, K., Matsuoka, M., Ogawara, Y., Suzuki, K., Tanaka, Y., Shibazaki, N., Hirano, T., 1984, PASJ, 36, 741

Nakahira, S., Shidatsu, M., Makishima, K., Ueda, Y., Yamaoka, K., Mihara, T., Negoro, H., Kawase, T., Kawai, N., Morita, K., 2018, PASJ, subm., arXiv: 1804.00800

Negoro, H., Ishikawa, M., Ueno, S., et al., 2017a, ATEL 10699

Negoro, H., Kawase, T., Sugizaki, M., et al., 2017b, ATEL 10708

Park, S. Q., Miller, J. M., McClintock, J. E., et al., 2004, ApJ, 610, 378

Ponti, G., Fender, R., Begelman, M., Dunn, R., Neilsen, J., Coriat, M., 2012, MNRAS, 422, L11

Reynolds, C., \& Fabian, A. C., 2008, ApJ, 675, 1048

Russell, T. D., Miller-Jones, J. C. A, Sivakoff, G. R., Tetarenko, A. J., 2017, ATEL 10711

Schnittman, J. D., Homan, J., \& Miller, J. M., 2006, ApJ, 642, 420

Shafee, R., Narayan, R., \& McClintock, J., 2008, ApJ, 676, 549

Sobczak, G., McClintock, J., Remillard, R., Bailyn, C., Orosz, J., 1999, ApJ, 520,776

Tomsick, J. A., Nowak, M. A., Parker, M., et al., 2014, ApJ, 780, 78

Wilkins, D., \& Fabian, A., 2012, MNRAS, 424, 1284

Wilms, J., Allen, A., \& McCray, R., 2000, ApJ, 542, 914

Xu, Y., Harrison, F., Garcia, J., et al., 2018, ApJ, 852, L34 
A NICER Look at MAXI J1535-571

TABLE 1

SPECTRAL FITTING RESULTS

\begin{tabular}{lll} 
Component & Parameter & Value \\
\hline tbabs & $N_{\mathrm{H}, \mathrm{ISM}}$ & $4.05(5) \times 10^{22} \mathrm{~cm}^{-2}$ \\
\hline diskbb & kT & $2.16(4) \mathrm{keV}$ \\
- & Norm. & $31_{-11}^{+6}$ \\
\hline relline & $\mathrm{E}$ & $6.72(4) \mathrm{keV}$ \\
- & $q_{\text {in }}$ & $2.65_{-0.65}^{1.35}$ \\
- & $q_{\text {out }}$ & $=q_{\text {in }}$ \\
- & $r_{\text {break }}$ & $6.0^{*} \mathrm{GM} / \mathrm{c}^{2}$ \\
- & $r_{\text {in }}$ & $144_{-60}^{+140} \mathrm{GM} / \mathrm{c}^{2}$ \\
- & $r_{\text {out }}$ & $990^{*} \mathrm{GM} / \mathrm{c}^{2}$ \\
- & $\theta$ & $37_{-13}^{+22} \mathrm{deg}$. \\
- & Norm. & $4.5(6) \times 10^{-3}$ \\
- & $a=c J / G M^{2}$ & $0.994(2)$ \\
\hline relxill & $q_{\text {in }}$ & $7.8(4)$ \\
- & $q_{\text {out }}$ & $1.40_{-0.19}^{+0.08}$ \\
- & $r_{\text {break }}$ & $5.8(2) \mathrm{GM} / \mathrm{c}^{2}$ \\
- & $r_{\text {in }}$ & $1.08(8) r_{I S C O}$ \\
- & $r_{\text {out }}$ & $990^{*} \mathrm{GM} / \mathrm{c}^{2}$ \\
- & $\quad$ & $67.4(8) \mathrm{deg}$. \\
- & $\Gamma$ & $2.303(5)$ \\
- & $\log (\xi)$ & $3.15(2)$ \\
- & $A_{\text {Fe }}$ & $0.62(2)$ \\
- & $f_{\text {refl }}$ & $2.65_{-0.18}^{+0.05}$ \\
- & $E_{\text {cut }}$ & $300^{*} \mathrm{keV}$ \\
- & Norm. & $0.179(5)$ \\
- & $\mathrm{E}$ & $8.98(3) \mathrm{keV}$ \\
\hline edge & $\tau$ & $5.2(5) \times 10^{-2}$ \\
- & Flux $(2.3-10 \mathrm{keV})$ & $4.5(1) \times 10^{-8} \mathrm{erg} \mathrm{cm}^{-2} \mathrm{~s}^{-1}$ \\
\hline Total & Unabs. flux $(0.5-10 \mathrm{keV})$ & $1.47(4) \times 10^{-7} \mathrm{erg} \mathrm{cm}^{-2} \mathrm{~s}^{-1}$ \\
Total & & \\
\hline & &
\end{tabular}

NOTE. - The parameters of the best-fit spectral model for MAXI J1535-571. In XSPEC parlance, the model is $t b a b s \times($ diskbb + relline + relxill $) \times e d g e$. The model returns $\chi^{2} / \nu=944.3 / 747$, where $\nu$ is the number of degrees of freedom. Quoted errors reflect the value of a given parameter on its $1 \sigma$ confidence limit. Symmetric errors in the last digit are quoted using parentheses. Frozen parameters are marked with an asterisk. For reference, a flux of $F \simeq 2 \times 10^{-8} \mathrm{erg} \mathrm{cm}^{-2} \mathrm{~s}^{-1}$ is equivalent to $1 \mathrm{Crab}$ in the $2-10 \mathrm{keV}$ band. Please see the text for additional details. 


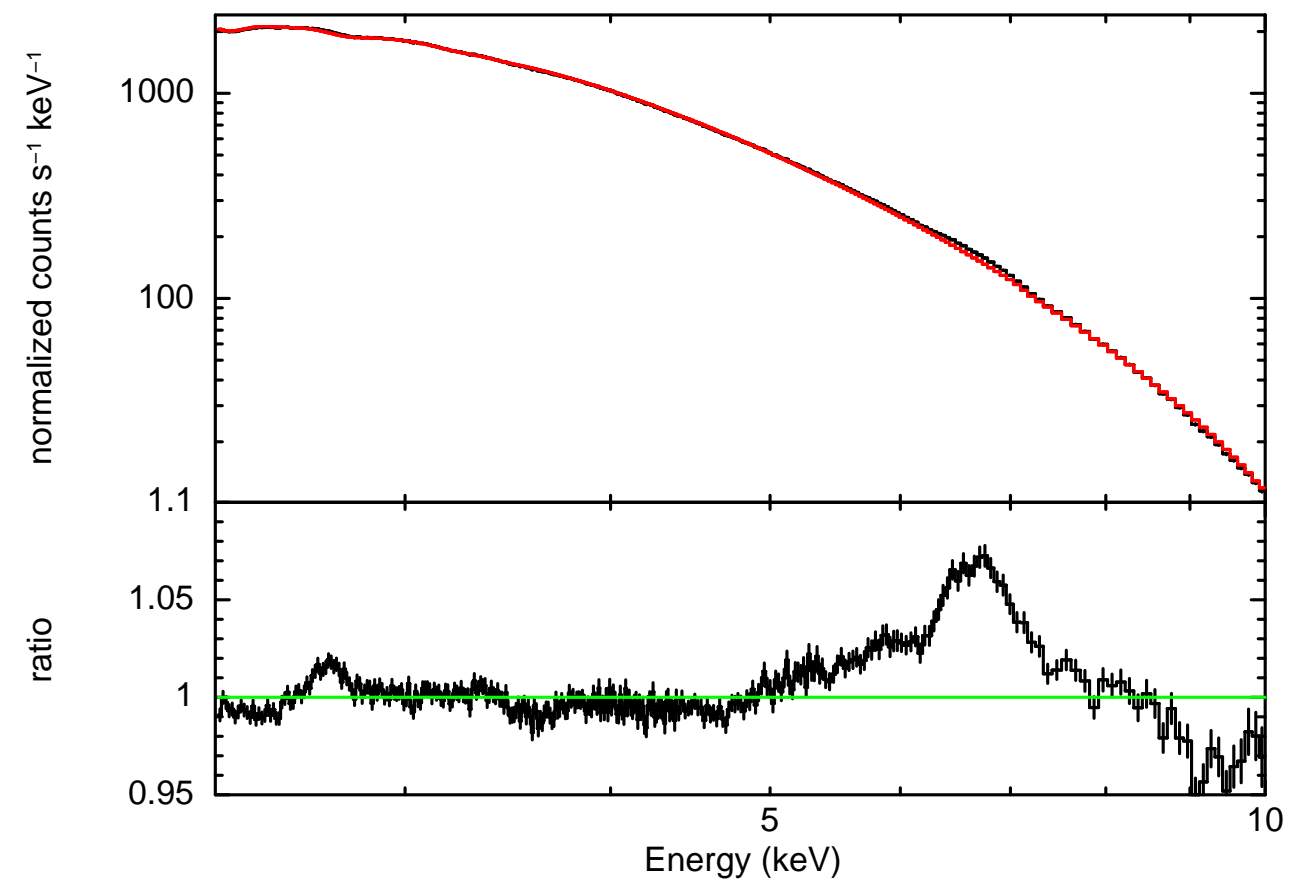

FIG. 1. - The spectrum of MAXI J1535-571, fit with a simple disk blackbody plus power-law model. The data are binned for visual clarity only, and the 4-7 keV was ignored in constructing the continuum fit. The data/model ration clearly reveals features consistent with strong, relativistically-skewed reflection from the inner accretion disk. Fits with relxill (e.g., Dauser et al. 2014, Garcia et al. 2014) measure a very high spin parameter, $a=c J / G M^{2}=0.994(2)(1 \sigma$ statistical errors only). Please see the text and Table 1 for additional details. 


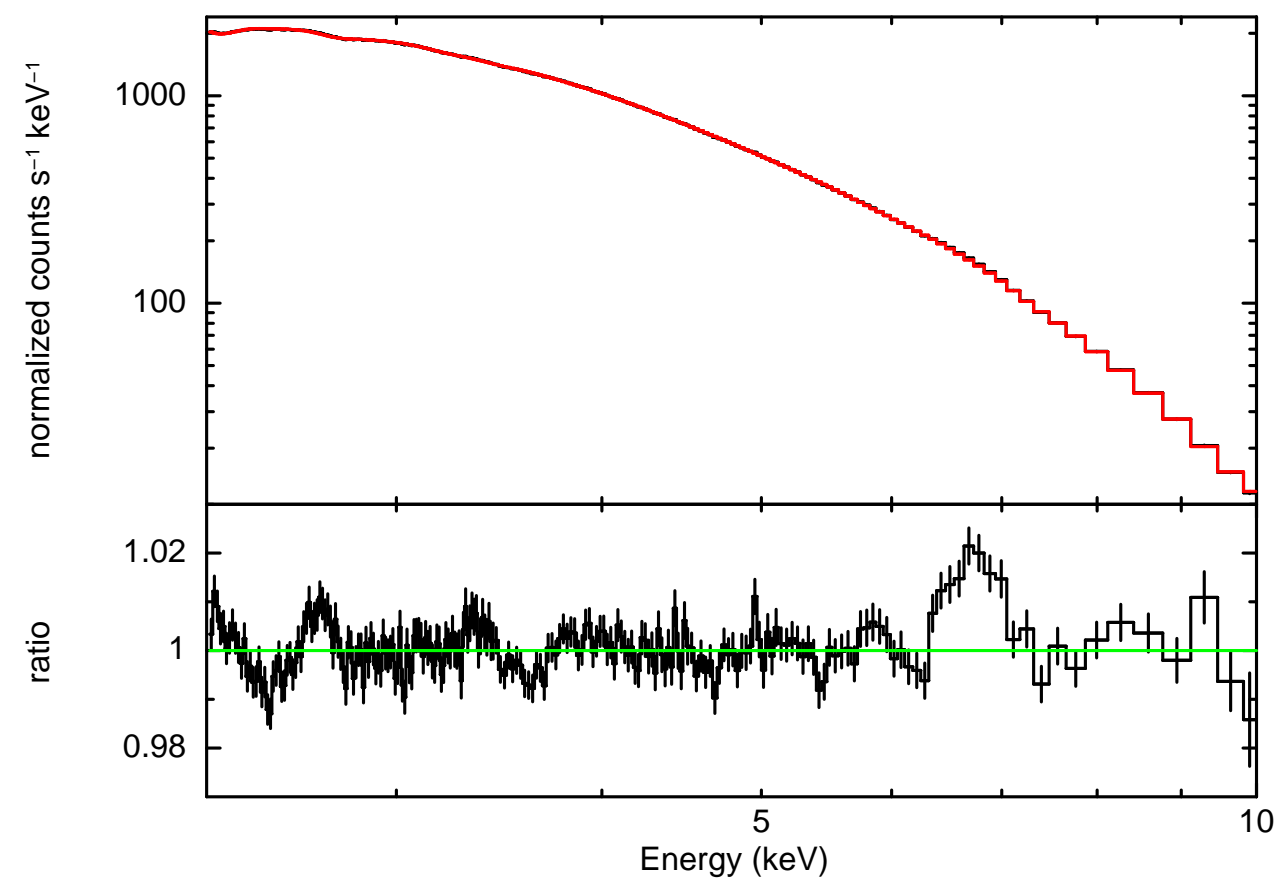

FIG. 2. - The best-fit model for MAXI J1535-571 is shown. The data have been rebinned for visual clarity only. The model includes blurred reflection from the inner disk, and a relatively narrow emission line that can be fit with a line originating at $r_{i n}=144_{-60}^{+140} \mathrm{GM} / \mathrm{c}^{2}$. The normalization of the narrow line has been set to zero in this plot to illustrate its nature. Please see the text and Table 1 for additional details. 
J. M. Miller et al.
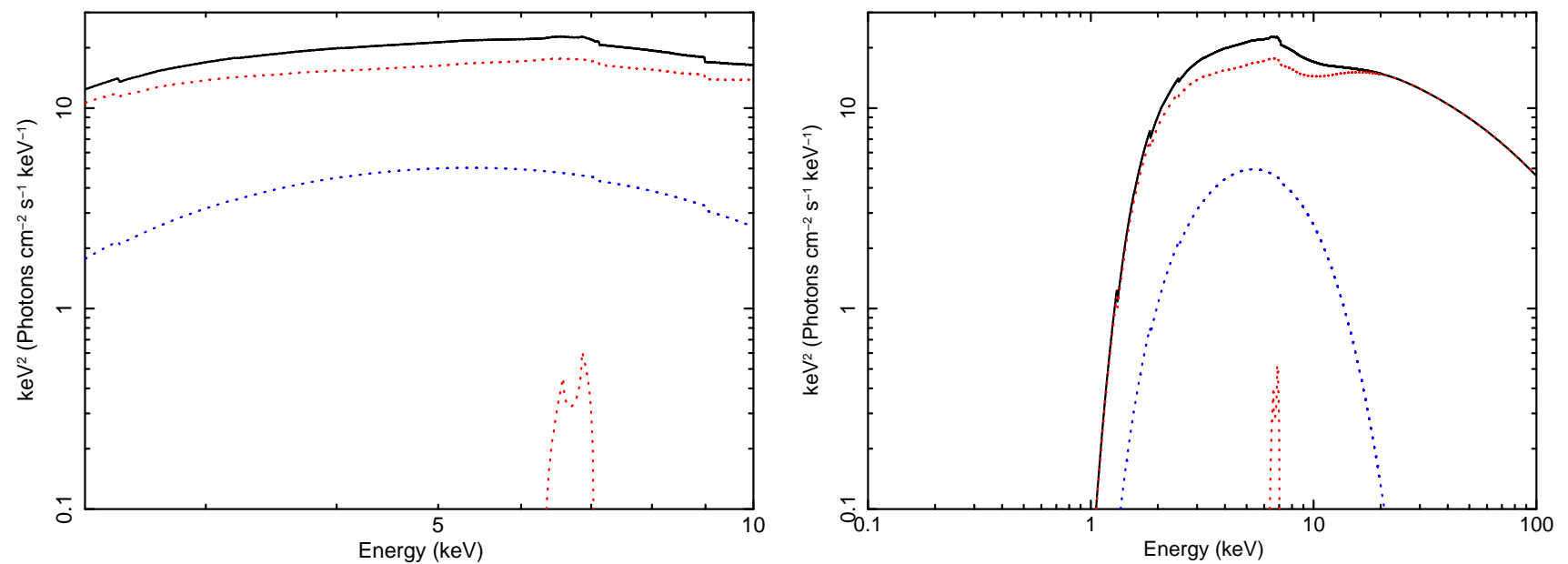

FIG. 3.- LEFT: The best-fit model for MAXI J1535-571 is shown here without the data, in the passband of the fit. The total model is shown in black; extremely blurred reflection from the innermost disk (including an incident power-law) and a separate, mildly relativistic line are shown in red; and the disk component is shown in blue. RIGHT: The best-fit model, extrapolated over a larger range. Please see the text and Table 1 for additional details. 